\title{
Interference of Skp2 effectively inhibits the development and metastasis of colon carcinoma
}

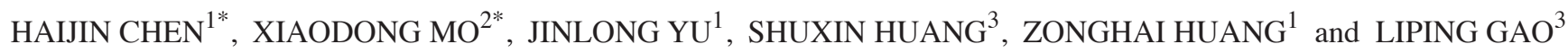 \\ ${ }^{1}$ Department of General Surgery, Zhujiang Hospital, Southern Medical University, Guangzhou, Guangdong 510280; \\ ${ }^{2}$ Department of Gastrointestinal Surgery, PLA No. 101 Hospital, Wuxi, Jiangsu 214044; ${ }^{3}$ Department of Ophthalmology, \\ Zhujiang Hospital, Southern Medical University, Guangzhou, Guangdong 510280, P.R. China
}

Received September 30, 2013; Accepted April 14, 2014

DOI: $10.3892 / \mathrm{mmr} .2014 .2308$

\begin{abstract}
Colon cancer is a common type of malignancy in the digestive system. The aim of the present study was to investigate the role of S-phase kinase-associated protein 2 (Skp2) in colon carcinoma and to identify whether depletion of Skp2 by Skp2-RNA interference (RNAi) attenuates the proliferation and migration of colon carcinoma. Three pairs of small interfering (si)RNA were designed based on the Skp2 gene sequence and the most effective one was used to silence the Skp2 gene in SW620 cells. Subsequent to the interference, quantitative polymerase chain reaction and western blot analysis were used for detecting the expression of Skp-2 mRNA and protein, respectively. The data demonstrated that the Skp2-siRNA effectively inhibited proliferation $(\mathrm{P}<0.01)$, increased the levels of apoptosis and induced $\mathrm{G}_{0} / \mathrm{G}_{1}$ phase arrest of the SW620 cells $(\mathrm{P}<0.01)$. Transfection of the Skp2 siRNA into SW620 cells effectively reduced Skp2 protein levels, while p27 protein levels increased. In the in vivo experiments, a lentiviral vector of the Skp2-RNAi transfected into SW620 cells markedly inhibited Skp2 expression, as detected by immunohistochemical analysis of nude mice. Additionally, tumorigenicity experiments showed that inhibition of Skp2 significantly increased the survival rate of nude mice. Thus, the in vitro and in vivo results demonstrated that interference of Skp2 expression significantly inhibited the proliferation and induced the apoptosis of SW620 cells. This suggests that Skp2 protein has an important role in the progression of colon cancer. Therefore, Skp2 may enable the
\end{abstract}

Correspondence to: Dr Jinlong Yu, Department of General Surgery, Zhujiang Hospital, Southern Medical University, 253 Industrial Avenue, Guangzhou, Guangdong 510280, P.R. China E-mail: jinlongyuwork@163.com

Miss Shuxin Huang, Department of Ophthalmology, Zhujiang Hospital, Southern Medical University, 253 Industrial Avenue, Guangzhou, Guangdong 510280, P.R. China

E-mail: huang-shuxin1967@163.com

*Contributed equally

Key words: S-phase kinase-associated protein 2, colon cancer, cell cycle, proliferation early diagnosis of colon cancer and provide new insights into molecular targets for cancer therapy.

\section{Introduction}

Colon cancer is a common type of malignancy in the gastrointestinal tract of the digestive system (1-4). Colon cancer is currently the fifth most common cause of cancer mortalities in China, mainly due to the recurrence of tumor metastasis $(5,6)$. The incidence of colorectal cancer has exhibited a clear positive association with rich blood supply, rapid growth, high levels of infiltration and propensity to metastasize (7-10). Numerous studies have focused on the pathogenesis and development of colon cancer (11). With the development of molecular biology techniques, much of the progress in the understanding of colon cancer that has occurred concerns the molecular mechanisms of colon cancer (12). Colorectal cancer is a disease originating from the epithelial cells lining the colon or rectum of the gastrointestinal tract. Benign adenoma initially develops from epithelial hyperplasia in normal colonic mucosa and then the potentially invasive and metastatic colon cancer forms (13). All of the progress that has occurred in the molecular mechanisms of colon cancer concerns the activation of oncogenes and inactivation of the tumor suppressor genes (14-16). Although much progress on the molecular mechanisms of colon cancer has been achieved, the molecular events remain to be fully elucidated.

Cell cycle regulation is critical for cell proliferation and tumorigenesis $(17,18)$. The cell cycle is regulated by various factors, including cyclin, cyclin-dependent kinases (CDKs) and CDK interacting protein (cip)/kinase inhibitory protein (kip). S-phase kinase-associated protein 2 (Skp2) mainly induces the degradation of CDK inhibitors, including p21 ${ }^{\text {cip1 }}$, p2 $7^{\mathrm{kip} 1}$ and p57 ${ }^{\mathrm{kip} 2}$ (19-21). As an F-box protein, Skp2 is a key regulator for cell cycle progression. The expression levels of Skp2 are low in the $G_{0} / G_{1}$ phase, while they are elevated in $S$ phase. The overexpression of the Skp2 gene may result in loss of control of the cells at the $\mathrm{G}_{1} / \mathrm{S}$ checkpoint, which could induce the cells to continuously proliferate and divide. Thus, Skp2 is a tumor-promoting factor. A number of studies have revealed that the overexpression of Skp2 is associated with the progression of a variety of types of human cancer (22-25). Functional deletion of Skp2 leads to stabilization of CDK inhibitors, which can subsequently induce cell-cycle delay or arrest $(26,27)$. However, 
the role of Skp2 expression in the metastasis and prognosis of colon cancer remains controversial.

The aim of the study was to explore the role of Skp2 in colon carcinoma and to identify whether depletion of Skp2 by Skp2 RNA interference attenuates the proliferation and migration of colon carcinoma.

\section{Materials and methods}

Cell lines and small interfering (si)RNA. The SW620 colon cell line (American Type Culture Collection, Rockville, MD, USA) was grown in Dulbecco's modified Eagle's medium with $100 \mathrm{mM}$ L-glutamine, $10 \%$ fetal bovine serum and $1 \%$ penicillin/streptomycin. Skp2-siRNA and scramble siRNA were synthetized by Jima Corporation (Shanghai, China). Skp2 p45 shRNA (h) Lentiviral Particles were purchased from Santa Cruz Biotechnology, Inc. (Santa Cruz, CA, USA).

Transfection. Cells were incubated in six-well plates $\left(3 \times 10^{5}\right.$ cells/well) overnight and were then transfected with siRNA using Lipofectamine 2000 (Invitrogen Life Technologies, Carlsbad, CA, USA) at a final RNA concentration of $100 \mathrm{nM}$, according to the manufacturer's instructions. The sequence of the Skp2-siRNA was as follows: Complementary oligonucleotides targeting Skp2, 5'-AGCTTTTCCAAAAAAGGGAGTGACAAAGACTTTG TCTCTTGAACAAAGTC-TTTGTCACTCCCG-3' and 5'-GA TCCGGGAGTGACAAAGACTTTGTTCAAGAGACAAAG TCTTTGTCACTCCCTTTTTTGGAAA-3'.

Western blot analysis. Whole cell extracts were prepared and separated by PAGE as previously described (28-30). The antibodies used included anti-Skp2 (Santa Cruz Biotechnology, Inc.), anti-p27 (Santa Cruz Biotechnology, Inc.), anti- $\beta$-actin and horseradish peroxidase-conjugated goat anti-mouse secondary antibody (Santa Cruz Biotechnology, Inc.) and were detected with an Enhanced Chemiluminescence Detection kit (Amersham Pharmacia Biotech, Amersham, UK).

Flow cytometric analysis. Annexin V-fluorescein isothiocyanate staining was used for a cell apoptosis assay as previously described (31). Propidium iodide (PI) staining was performed to analyze cell cycle progression as previously described (32). Briefly, $1 \times 10^{6}$ colon cancer cells were washed three times in cold phosphate-buffered saline (PBS) and fixed in 4\% paraformaldehyde for 30 min. Following two further washes in PBS, PI and RNase A (Sigma-Aldrich, St. Louis, MO, USA) were added to a final concentration of $100 \mathrm{ng} / \mathrm{ml}$ each. After incubation for $15 \mathrm{~min}$ at room temperature, the cells were analyzed by flow cytometry (FACScan; BD Biosciences, Erembodegem, Belgium).

Scratch assay. The scratch assay was used to measure basic cell migration parameters. Briefly,cells were grown to confluence and a thin 'wound' was introduced by scratching with a pipette tip. The distance of which the cells at the wound edge had migrated into the wound space was measured following 0 and $12 \mathrm{~h}$.

MTT assay. MTT assay kits were purchased from Sigma-Aldrich. The colon cancer cells were seeded in 48-well plates. After $6 \mathrm{~h}$, the cells were transfected with siRNA specific for Skp2 or
A

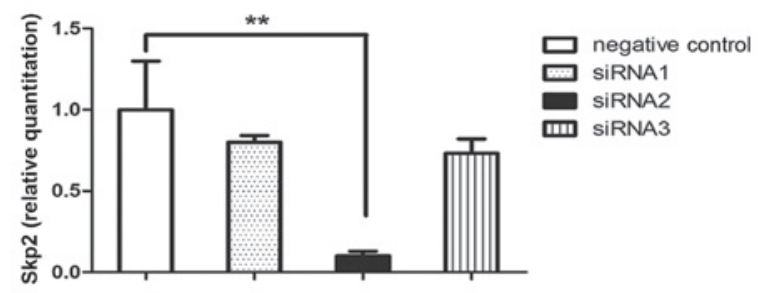

B control siRNA1 siRNA2 siRNA3

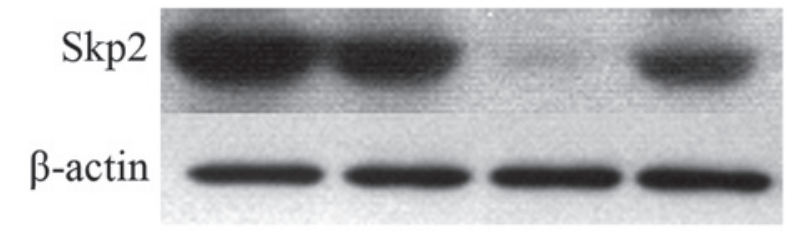

Figure 1. Identification of the most effective siRNA of the Skp2 gene in SW620 cells. The cells were plated in 48-well plates and three pairs of Skp2-siRNA were transfected into the SW620 cells. The expression levels of the Skp2 gene were detected $48 \mathrm{~h}$ later by (A) quantitative polymerase chain reaction and (B) western blot analysis. $\beta$-actin was used an internal reference. Results are presented as the mean \pm standard error of the mean. ${ }^{* *} \mathrm{P}<0.01$, compared with the control group. Skp2, S-phase kinase-associated protein 2; si, small interfering.

scramble siRNA for different time periods. The MTT solution was added to the cells (10\% of total volume) and after a period of $4 \mathrm{~h}$, the media was removed and replaced with acidified isopropanol and then the absorbance was read at $490 \mathrm{~nm}$.

Animals and grouping. Male BALB/c (nu/nu) mice were obtained from the Guangdong Medical Laboratory Animal Center (Guangzhou, China) and housed under specific pathogen-free conditions. The mice were kept in a 12-h light and dark cycle. All animals were randomly divided into three groups (group A, control; group B, the group transfected with the lentiviral vector of Skp2-RNA interference (RNAi); and group C, scrambled siRNA group) and each group contained ten mice. All procedures were in accordance with the Declaration of Helsinki of the World Medical Association. The protocols were also approved by the Institutional Animal Care and Use Committee of Zhujiang Hospital of Southern Medical University (Guangzhou, China). The survival days were recorded and the survival rates were obtained using GraphPad Prism 5 (GraphPad Software, Inc., La Jolla, CA, USA).

Statistical analyses. Data were entered into a database and analyzed using SPSS software (SPSS, Inc., Chicago, IL, USA). The comparison of Skp2 and p2 $7^{\text {kipl }}$ mRNA expression following different treatments was conducted using a Student's $\mathrm{t}$-test. Results are presented as the mean \pm standard error of the mean. $\mathrm{P}<0.01$ was considered to indicate a statistically significant difference.

\section{Results}

Skp2-siRNA2 is the most effective sequence for interfering with the Skp2 gene. To study the role of Skp2 as a therapeutic target for the treatment of colon cancer cells, three pairs of interfering RNAs (siRNA) were designed to specifically silence endogenous Skp2 expression and were transfected into SW620 cells. The expression levels of Skp2 were detected $48 \mathrm{~h}$ later by 
A

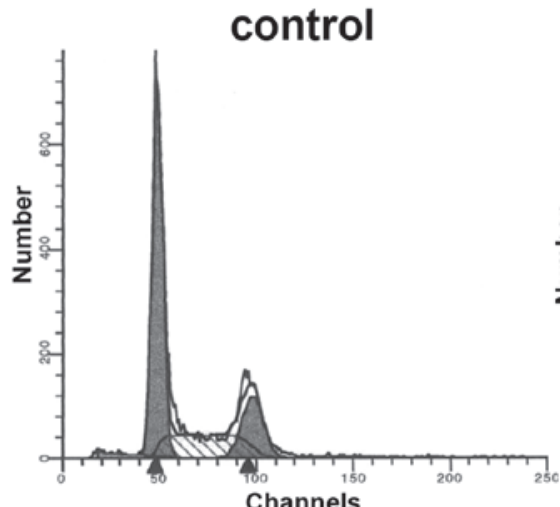

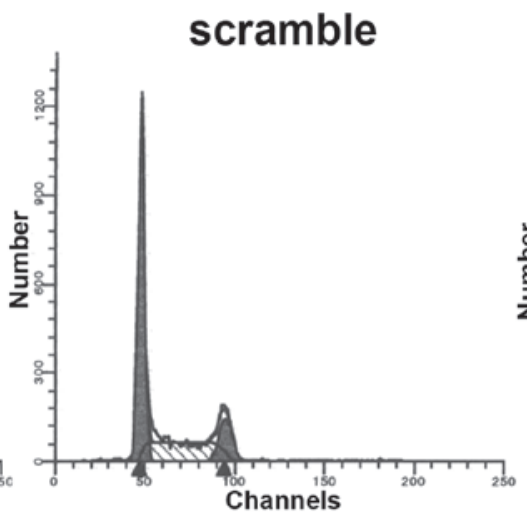

scramble

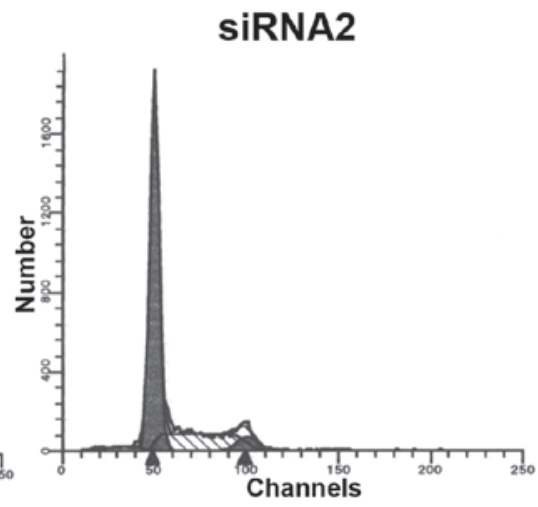

B
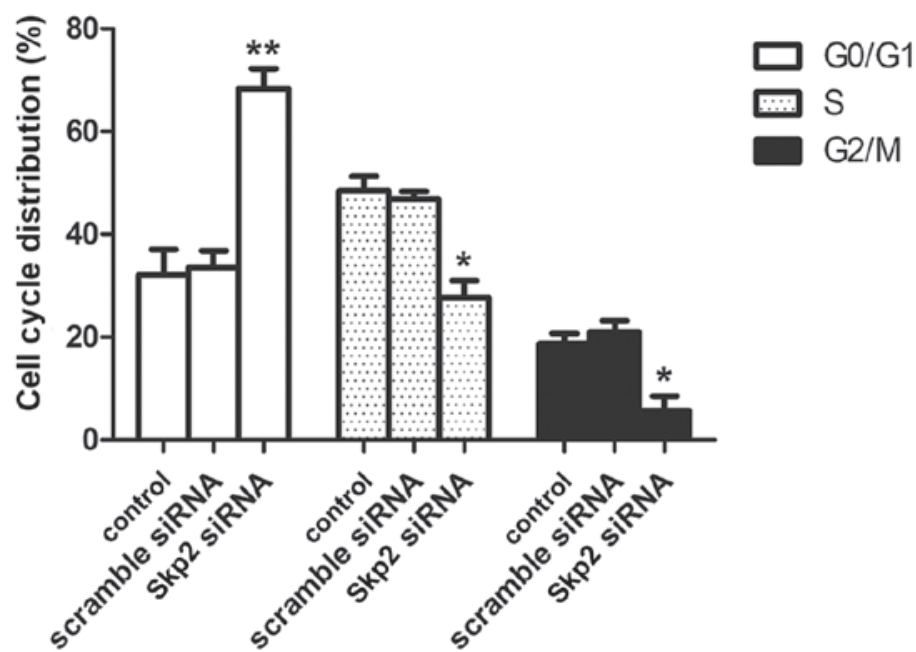
with Skp2-siRNA or scrambled siRNA. (A) The proportion of the cells in each phase was determined by flow cytometry of propidium iodide-stained cells and (B) the data were quantified. Results are presented as the mean \pm standard error of the mean. ${ }^{*} \mathrm{P}<0.05$ and ${ }^{* *} \mathrm{P}<0.01$, compared with the respective control group. si, small interfering; Skp2, S-phase kinase-associated protein 2.

quantitative polymerase chain reaction and $\beta$-actin was used as the positive control in the experiment. The results demonstrated that the Skp2-siRNA2 sequence was the most effective at silencing Skp2 expression (Fig. 1A). This result was consistent with the results detected by western blot analysis (Fig. 1B).

Skp2-siRNA induces cell cycle arrest in $G_{0} / G_{l}$ phase. In order to elucidate the mechanism of Skp2 siRNA, cell cycle analysis of SW620 cells was performed. As shown in Fig. 2, the data demonstrated an accumulation of colon cancer cells in $\mathrm{G}_{0} / \mathrm{G}_{1}$ phase, with a relative paucity of cells traversing through the $S$ and $\mathrm{G}_{2} / \mathrm{M}$ phases compared with those in the control groups.

Skp2-RNAi inhibits the migratory ability of SW620 cells. To determine the role of endogenously expressed Skp2 in the regulation of SW620 cell migration and proliferation, the in vitro scratch assay was used to measure cell migration. As shown in Fig. 3, the number of migrated SW620 cells was clearly reduced following transfection with Skp2-RNAi, compared with that of the control and the group transfected with scramble siRNA groups.

Transfection with Skp2-siRNA induces apoptosis of SW620 cells. Subsequently, whether treatment of cancer cells with
siRNA specific for Skp2 was able to further induce apoptosis was investigated. As shown in Fig. 4, the apoptotic rates of colon cells transfected with Skp2-siRNA were significantly higher compared with those of the cells transfected with scrambled siRNA $(38.90 \pm 4.5 \%$ for the Skp2-siRNA group compared with $8.2 \pm 1.8 \%$ for the scrambled siRNA group, $\mathrm{n}=10 ; \mathrm{P}=0.0039$ ).

Skp2-RNAi inhibits cell growth. In order to detect the effect of Skp2-siRNA on cell growth, an MTT assay kit was used to evaluate the proliferation of SW620 cells. As shown in Fig. 5, the optical density ${ }_{490}$ values in the Skp2 siRNA group were significantly lower than the values in the control and scramble siRNA groups $(\mathrm{P}<0.01)$, which suggested that cell growth was significantly inhibited along with the downregulation of Skp2 expression levels.

Reducing the expression levels of Skp2 increases $p 27^{k i p 1}$ expression levels in SW620 cells. In the present study, SW620 cells were depleted of endogenous Skp2 by RNAi with siRNA specific for Skp2 mRNA. The Skp2-depleted cells exhibited increased levels of endogenous p27 (Fig. 6). $\beta$-actin was used as an internal reference. In parallel, the results demonstrated that the Skp2-mediated degradation of $\mathrm{p} 27^{\mathrm{kip} 1}$ had an important role in cell proliferation and survival. 


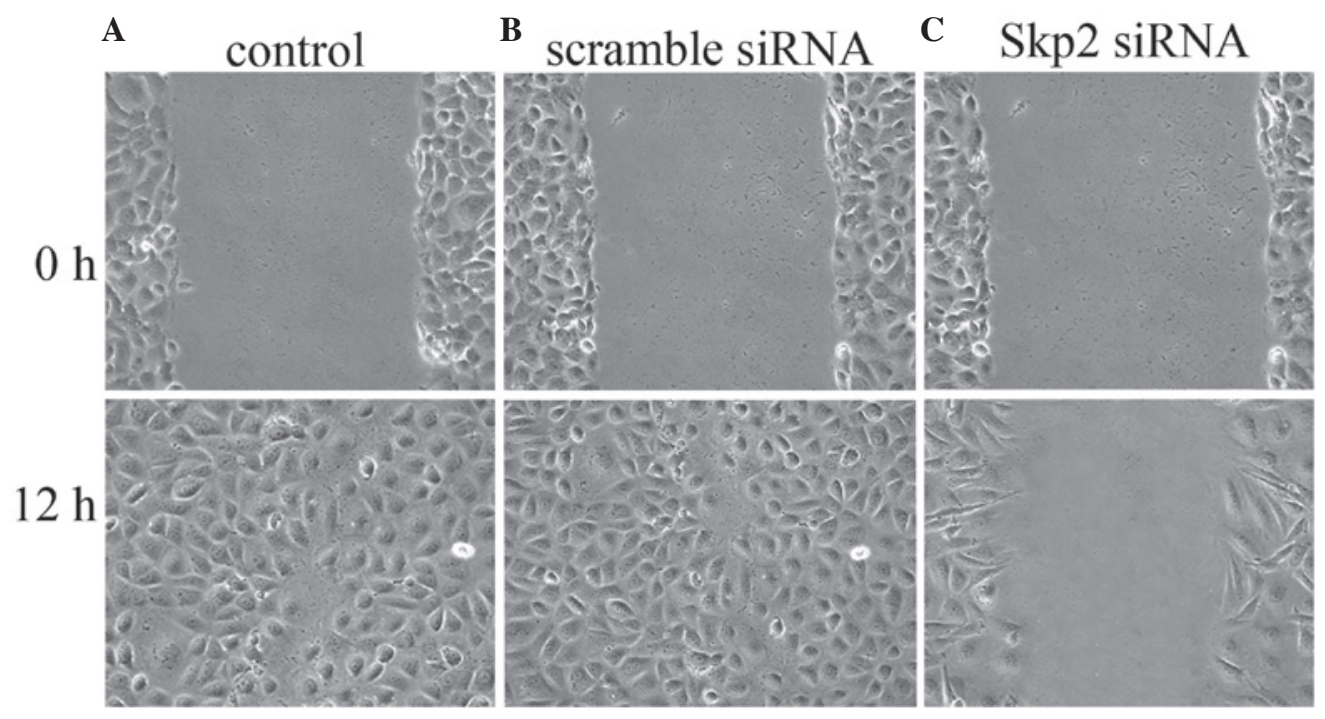

Figure 3. In vitro scratch assay. (A) Untreated group, (B) SW620 colon cancer cells treated with scrambled siRNA for $12 \mathrm{~h}$ after the scratch was performed and (C) SW620 cells transfected with Skp2-siRNA for $12 \mathrm{~h}$ after the scratch was performed. si, small interfering; Skp2, S-phase kinase-associated protein 2.

A

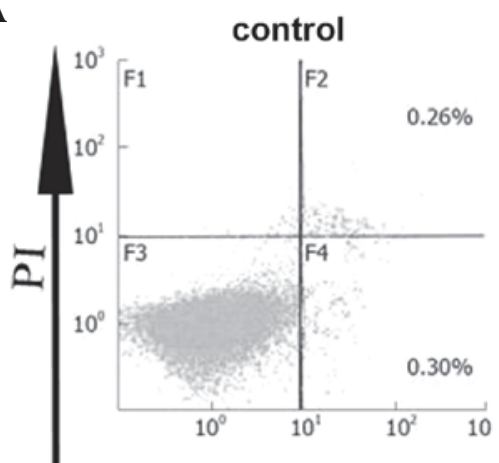

scramble

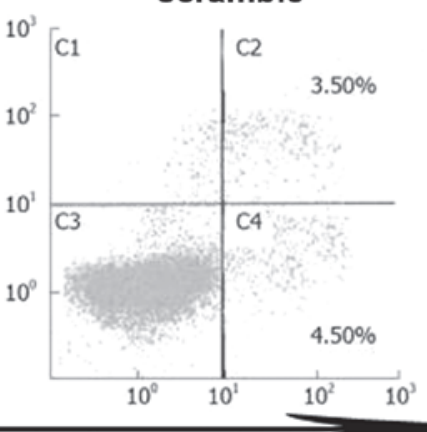

Skp2 siRNA2

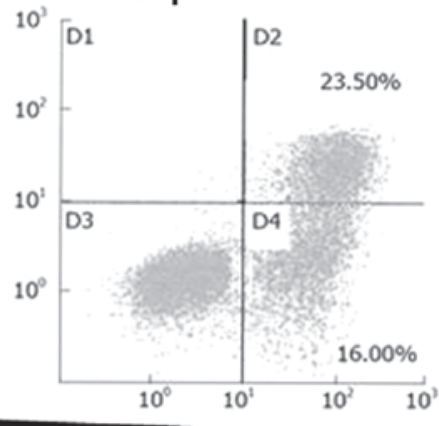

B

FITC

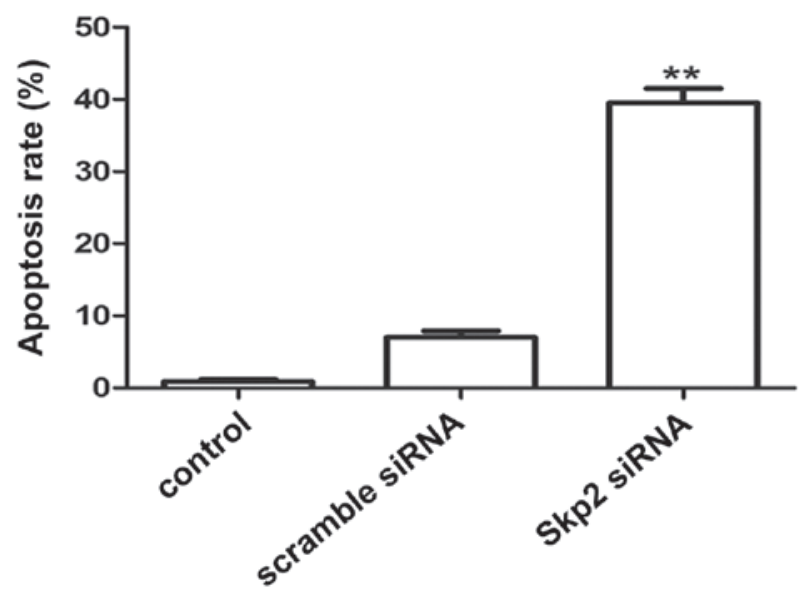

Figure 4. Induction of apoptosis was determined by PI-Annexin V staining. The cells were transfected with Skp2-siRNA and scrambled siRNA for $48 \mathrm{~h}$. PI-Annexin V-positive cells were analyzed by (A) fluorescence-activated cell sorting and (B) the percentage of the PI-Annexin V-positive cells was quantified and shown in the histograms. Results are presented as the mean \pm standard error of the mean. ${ }^{* *} \mathrm{P}<0.01$, compared with the control group or the group treated with scrambled siRNA. Skp2, S-phase kinase-associated protein 2; si, small interfering; PI, propidium iodide; FITC, fluorescein isothiocyanate.

Tumorigenicity experiments in nude mice. To further define the potential efficacy of Skp2-siRNA, a lentiviral vector of Skp2-RNAi was used and its activity against the proliferation and metastasis of colon carcinoma cells in a nude mouse model was evaluated. Paraffin-embedded samples were analyzed by immunohistochemical staining for Skp2 after challenging the animals with colon cancer cells for two weeks. The results revealed that Skp2-siRNA noticeably suppressed the expression of Skp2 in the tissues of nude mice (Fig. 7). Notably, the survival rates of the mice in the Skp2-RNAi group were 


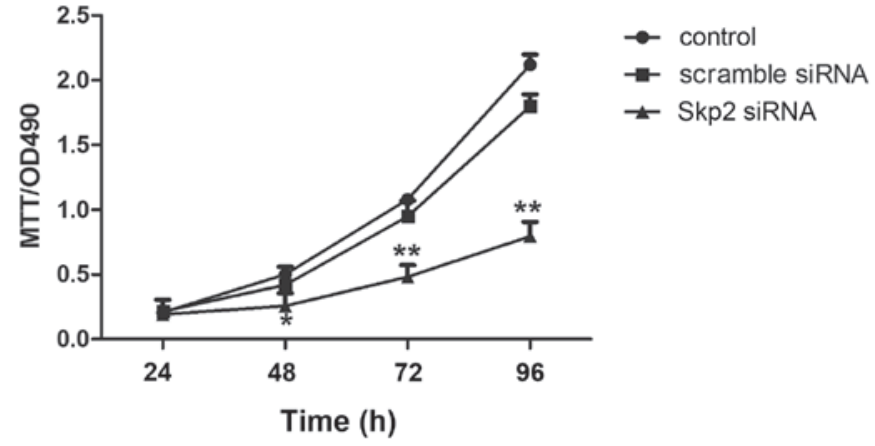

Figure 5. Inteference of Skp2 inhibited proliferation of SW620 cells, as detected by an MTT assay. SW620 cells $\left(1 \times 10^{5}\right)$ were planted in a 96-well plate and treated for different time periods in the presence of Skp2 siRNA or scrambled siRNA. The number of SW620 cells was measured. Untreated cells were used as the negative control. Data are shown as the mean \pm standard error of the mean of at least three independent experiments on different individuals. ${ }^{* *} \mathrm{P}<0.01$ compared with the control group or the group treated with scrambled siRNA. OD, optical density; si, small interfering; Skp2, S-phase kinase-associated protein 2.

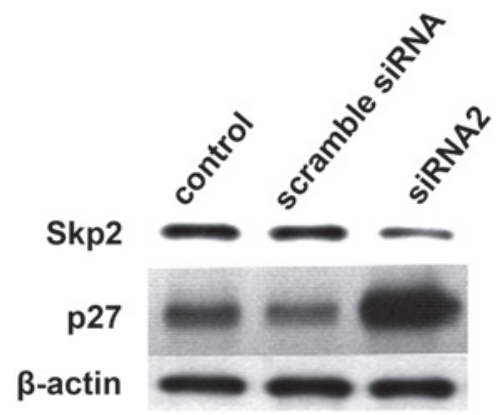

Figure 6. Expression levels of p27 $7^{\mathrm{kip} 1}$ were increased in SW620 cells. SW620 cells $\left(2 \times 10^{5}\right)$ were planted in a 24-well plate and transfected for $24 \mathrm{~h}$ in the presence of Skp2 siRNA or scrambled siRNA. Untreated cells were used as the negative control. Total cell lysates were analyzed for Skp2, p27 and $\beta$-actin by western blot analysis. $\beta$-actin was used as an internal reference. Skp2, S-phase kinase-associated protein 2; si, small interfering.

significantly higher than those in the scrambled siRNA and control groups ( $\mathrm{P}=0.003$ vs. scrambled group, $\mathrm{P}=0.006$ vs. control group) (Fig. 8).

\section{Discussion}

In the present study, three pairs of Skp2 siRNA were designed to inhibit the endogenous Skp2 expression in colon cancer cells. A specific Skp2-siRNA which effectively reduced the endogenous expression levels of the Skp2 gene was successfully selected. Interference of Skp2 expression significantly inhibited the proliferation of SW620 cells compared with those in the control group, as detected by an MTT assay, which was consistent with the results detected in other cell lines, including HCT116, DLD-1 and DU145 (data not shown). The in vitro scratch assay results showed that SW620 cell growth was reduced in the Skp2-siRNA group compared with that in the control group, suggesting that silencing of Skp2 markedly reduced cell migration and proliferation, which was consistent with the results of the MTT assay.

As previously mentioned, Skp2 is an F-box substrate-recognition subunit of the Skp-Cullin-F-box protein (SCF)
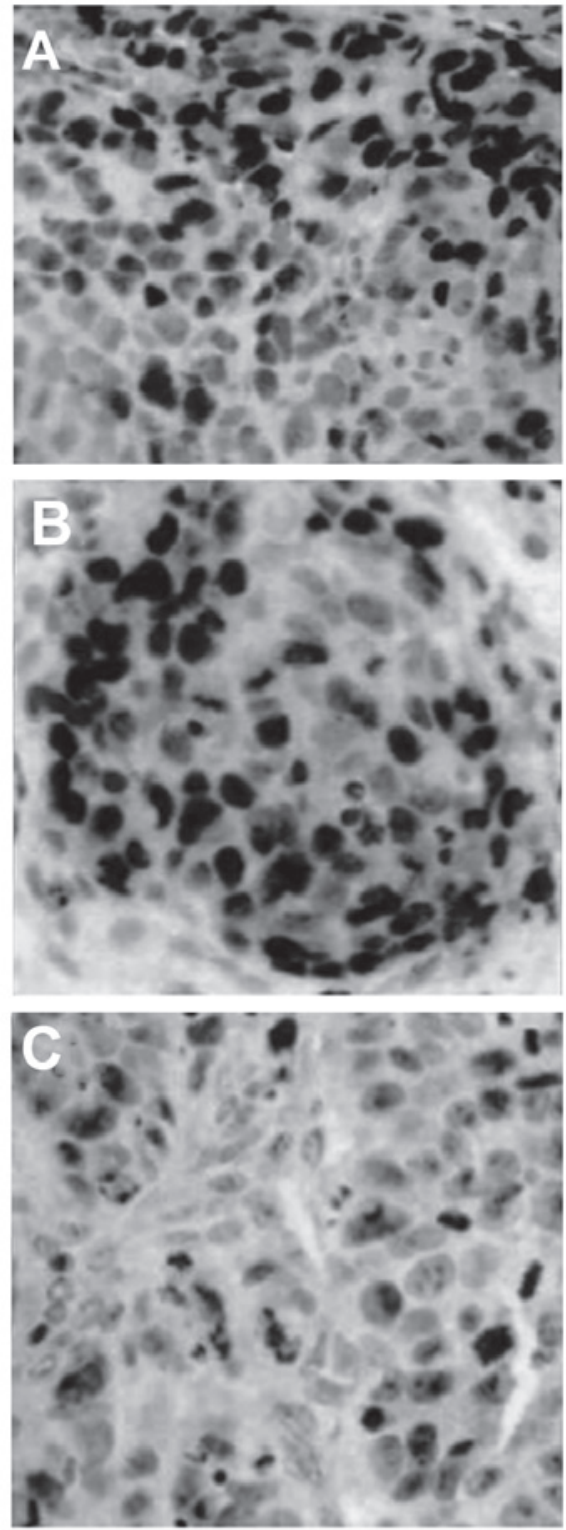

Figure 7. Paraffin-embedded samples were analyzed by immunohistochemical staining for Skp2. Skp2 protein was mainly located and expressed in the nucleus of the tumor cells. Skp2 expression in tumor tissues of the (A) control, (B) scrambled siRNA and (C) Skp2 siRNA groups (images of Skp2 staining were captured at $\mathrm{x} 400$ original magnification). Skp2, S-phase kinase-associated protein 2; si, small interfering.

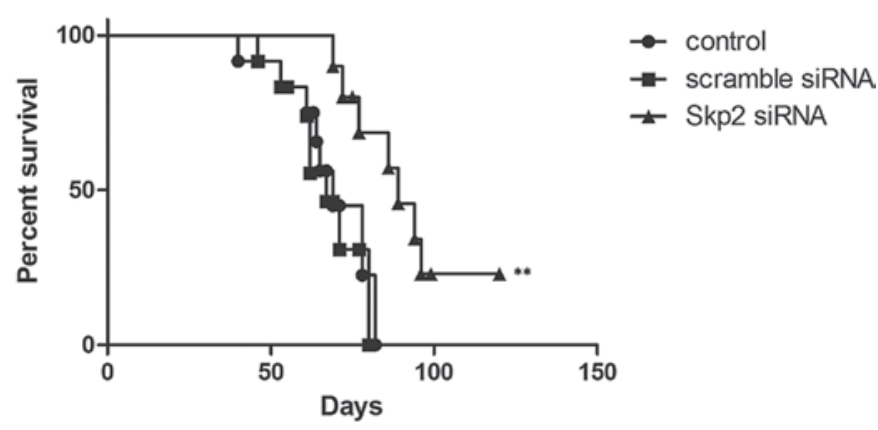

Figure 8. Survival rate of nude mice following lethal SW620 cell challenge. The nude mice were challenged subcutaneously with $5 \times 10^{5}$ SW620 colon cancer cells in the flank area. The survival rate at 29 days was determined as follows: $100 \mathrm{x}$ (number of survivors)/(number of challenged mice). Each group contained ten mice. ${ }^{* *} \mathrm{P}=0.003$ vs. scrambled group, $\mathrm{P}=0.006$ vs. control group. si, small interfering; Skp2, S-phase kinase-associated protein 2. 
ubiquitin-protein ligase complex which regulates the progression of the cell cycle by degrading the tumor suppressor gene $\mathrm{p} 27^{\mathrm{kip} 1}$ in a ubiquitin-mediated manner (17,18,33-35). In the present study, the effects Skp2 depletion by RNAi on cell cycle progression in colon cancer cells were also identified. The results demonstrated that loss of Skp2 resulted in a marked reduction in $\mathrm{G}_{0} / \mathrm{G}_{1}$ progression in colon cancer cells, whereas the number of cells in the $\mathrm{G}_{2} / \mathrm{M}$ phase was reduced compared with those in the control group. Thus, the cell cycle was blocked in $\mathrm{G}_{0} / \mathrm{G}_{1}$ phase, and this delay was accompanied by an accumulation of $\mathrm{p} 27^{\mathrm{kip} 1}$. Elevated levels of Skp2 are usually accompanied by reduced levels of $\mathrm{p} 27^{\mathrm{kip} 1}$, which are considered to be associated with highly aggressive tumors and a poor prognosis in various types of cancer. The results of the present study also revealed that regulation of colon carcinoma proliferation by Skp2-siRNA is dependent on $\mathrm{p} 27^{\mathrm{kip} 1}$ protein expression.

Studies have previously proposed targeting of E3 ligases as a rational strategy to inhibit the progression of cancer by inhibition of proteasomes $(36,37)$. Consistent with this theory, inhibition of SCF Skp2 in the present study blocked proliferation and migration of SW620 cells by inducing $\mathrm{G}_{0} / \mathrm{G}_{1}$ cell-cycle arrest and apoptosis. In addition to the in vitro inhibition of the proliferation and migration of SW620 cells, the antitumor effect of Skp2-RNAi on nude mice was also investigated in the present study through tumorigenicity experiments. All the results suggested that treatment with Skp2-RNAi represses the growth of metastatic tumors in vivo. Additionally, the immunohistochemical results demonstrated that a lentiviral vector of Skp2-RNAi effectively inhibited Skp2 expression in a murine model.

Thus, the results of the present study confirmed the hypothesis that Skp2 siRNA may be a useful therapeutic protocol for the treatment of colon carcinoma. Future studies may gradually elucidate the mechanism of Skp2 in colon carcinoma, and Skp2 may enable the early diagnosis of colon cancer and provide new insight into the molecular targets for cancer therapy.

\section{References}

1. Leufkens AM, van den Bosch MA, van Leeuwen MS and Siersema PD: Diagnostic accuracy of computed tomography for colon cancer staging: a systematic review. Scand J Gastroenterol 46: 887-894, 2011.

2. Merika E, Saif MW, Katz A, Syrigos K and Morse M: Review. Colon cancer vaccines: an update. In Vivo 24: 607-628, 2010.

3. Sharif S and O'Connell MJ: Gene signatures in stage II colon cancer: a clinical review. Curr Colorectal Cancer Rep 8: 225-231, 2012.

4. Galfrascoli E, Piva S, Cinquini M, et al; ORION Collaborative Group: Risk/benefit profile of bevacizumab in metastatic colon cancer: a systematic review and meta-analysis. Dig Liver Dis 43: 286-294, 2011

5. Hu NC, Hsieh SC, Chen TJ and Chang JY: Multiple primary malignancies including colon, stomach, lung, breast, and liver cancer: a case report and literature review. Chin Med J (Engl) 122: 3091-3093, 2009.

6. Zhao Z, Wei D, Mu Y, et al: Mutational analysis of SKP2 and P27 in Chinese Han women with premature ovarian failure. Reprod Biomed Online 27: 104-106, 2013.

7. Wang RH, Xie JG, Chen H, Ren TT and Zhang YC: Expression of angiopoietin-2 and vascular endothelial growth factor in human colon cancer. Nan Fang Yi Ke Da Xue Xue Bao 33: 1236-1239, 2013 (In Chinese).

8. Liu SJ, Yang XH, Ren JQ and Zhu XJ: Clinical significance of tumor budding detection in stage II (colon cancer). Zhonghua Wei Chang Wai Ke Za Zhi 16: 730-734, 2013 (In Chinese).
9. McPartland S, Hyman N, Blaszyk $\mathrm{H}$ and Osler T: The number of lymph nodes in colon cancer specimens: what do the numbers really mean? Colorectal Dis 12: 770-775, 2010.

10. Nesbakken A and Gaard M: Surgical treatment of colon cancer. Tidsskr Nor Laegeforen 127: 2942-2945, 2007 (In Norwegian).

11. Grady WM and Pritchard CC: Molecular alterations and biomarkers in colorectal cancer. Toxicol Pathol 42: 124-139, 2014.

12. Remo A, Pancione M, Zanella C and Vendraminelli R: Molecular pathology of colorectal carcinoma. A systematic review centred on the new role of the pathologist. Pathologica 104: 432-441, 2012.

13. Kuniyasu H, Ohmori H, Sasaki T, et al: Production of interleukin 15 by human colon cancer cells is associated with induction of mucosal hyperplasia, angiogenesis, and metastasis. Clin Cancer Res 9: 4802-4810, 2003.

14. Leng Z, Tao K, Xia Q, et al: Kruppel-like factor 4 acts as an oncogene in colon cancer stem cell-enriched spheroid cells. PLoS One 8: e56082, 2013.

15. Zhu G, Wang Y, Huang B, et al: A Rac1/PAK1 cascade controls $\beta$-catenin activation in colon cancer cells. Oncogene 31: 1001-1012, 2012.

16. Jia ZC, Wan YL, Tang JQ, et al: Tissue factor/activated factor VIIa induces matrix metalloproteinase-7 expression through activation of c-Fos via ERK1/2 and p38 MAPK signaling pathways in human colon cancer cell. Int J Colorectal Dis 27: 437-445, 2012.

17. Calvisi DF, Pinna F, Ladu S, et al: The degradation of cell cycle regulators by SKP2/CKS1 ubiquitin ligase is genetically controlled in rodent liver cancer and contributes to determine the susceptibility to the disease. Int J Cancer 126: 1275-1281, 2010.

18. Bashir T, Pagan JK, Busino L and Pagano M: Phosphorylation of Ser72 is dispensable for Skp2 assembly into an active SCF ubiquitin ligase and its subcellular localization. Cell Cycle 9: 971-974, 2010.

19. Bretones G, Acosta JC, Caraballo JM, et al: SKP2 oncogene is a direct MYC target gene and MYC down-regulates p27(KIP1) through SKP2 in human leukemia cells. J Biol Chem 286: 9815-9825, 2011.

20. Cen B, Mahajan S, Zemskova M, et al: Regulation of Skp2 levels by the Pim-1 protein kinase. J Biol Chem 285: 29128-29137, 2010.

21. Zhang B, Ji LH, Liu W, Zhao G and Wu ZY: Skp2-RNAi suppresses proliferation and migration of gallbladder carcinoma cells by enhancing p27 expression. World J Gastroenterol 19: 4917-4924, 2013.

22. Abdou AG, Asaad NY, Abd El-Wahed MM, Samaka RM and Allah MS: The prognostic value of Skp2 expression in Egyptian diffuse large B-cell lymphoma. Appl Immunohistochem Mol Morphol 20: 47-55, 2012.

23. Huang H, Zhao W and Yang D: Stat3 induces oncogenic Skp2 expression in human cervical carcinoma cells. Biochem Biophys Res Commun 418: 186-190, 2012.

24. Wang G, Chan CH, Gao Y and Lin HK: Novel roles of Skp2 E3 ligase in cellular senescence, cancer progression, and metastasis. Chin J Cancer 31: 169-177, 2012.

25. Xiao J, Yin S, Li Y, et al: SKP2 siRNA inhibits the degradation of P27kip1 and down-regulates the expression of MRP in HL-60/A cells. Acta Biochim Biophys Sin (Shanghai) 41: 699-708, 2009.

26. Kullmann MK, Grubbauer C, Goetsch K, et al: The p27-Skp2 axis mediates glucocorticoid-induced cell cycle arrest in T-lymphoma cells. Cell Cycle 12: 2625-2635, 2013.

27. Liu S and Yamauchi H: p27-Associated G1 arrest induced by hinokitiol in human malignant melanoma cells is mediated via down-regulation of $\mathrm{pRb}$, Skp2 ubiquitin ligase, and impairment of Cdk2 function. Cancer Lett 286: 240-249, 2009.

28. Nishitani H, Sugimoto N, Roukos V, et al: Two E3 ubiquitin ligases, SCF-Skp2 and DDB1-Cul4, target human Cdt1 for proteolysis. EMBO J 25: 1126-1136, 2006.

29. Peng L, Xu Z, Zhou Y, Yang T, Liang ZQ and Zhang M: Effect of rosiglitazone on cells cycle, apoptosis and expression of Skp2 and p27Kip1 in hepatocellular carcinoma cell line. Zhonghua Gan Zang Bing Za Zhi 18: 148-149, 2010 (In Chinese).

30. Schulman BA, Carrano AC, Jeffrey PD, et al: Insights into SCF ubiquitin ligases from the structure of the Skp1-Skp2 complex. Nature 408: 381-386, 2000.

31. Hussain AR, Khan AS, Ahmed SO, et al: Apigenin induces apoptosis via downregulation of S-phase kinase-associated protein 2-mediated induction of p27Kip1 in primary effusion lymphoma cells. Cell Prolif 43: 170-183, 2010. 
32. Herst PM, Broadley KW, Harper JL and McConnell MJ: Pharmacological concentrations of ascorbate radiosensitize glioblastoma multiforme primary cells by increasing oxidative DNA damage and inhibiting G2/M arrest. Free Radic Biol Med 52: 1486-1493, 2012.

33. Wei Z, Jiang X, Liu F, et al: Downregulation of Skp2 inhibits the growth and metastasis of gastric cancer cells in vitro and in vivo. Tumour Biol 34: 181-192, 2013.

34. Wu J, Lee SW, Zhang X, et al: Foxo3a transcription factor is a negative regulator of Skp2 and Skp2 SCF complex. Oncogene 32: $78-85,2013$.
35. Wu L, Grigoryan AV, Li Y, Hao B, Pagano M and Cardozo TJ: Specific small molecule inhibitors of Skp2-mediated p27 degradation. Chem Biol 19: 1515-1524, 2012.

36. Zhu L: Skp2 knockout reduces cell proliferation and mouse body size: and prevents cancer? Cell Res 20: 605-607, 2010.

37. Hsu JD, Kao SH, Ou TT, Chen YJ, Li YJ and Wang CJ: Gallic acid induces G2/M phase arrest of breast cancer cell MCF-7 through stabilization of $\mathrm{p} 27(\mathrm{Kip} 1)$ attributed to disruption of p27(Kip1)/Skp2 complex. J Agric Food Chem 59: 1996-2003, 2011. 